\title{
Intelligent and Personalised Hydrocephalus Treatment and Management
}

\author{
Lina Momani, Abdel Rahman Alkharabsheh and Waleed Al-Nuaimy \\ University of Liverpool \\ United Kingdom
}

\section{Introduction}

Personalised healthcare is primarily concerned with the devolution of patient monitoring and treatment from the hospital to the home. Solutions, such as body-worn sensors for clinical and healthcare monitoring, improve the quality of life by offering patients greater independence. Such solutions can go beyond monitoring to active intervention and treatment based on sensory measurement and patient feedback, effectively taking healthcare out of the hospital environment. Such personalised healthcare solutions play an increasingly important role in delivering high quality and cost-effective healthcare.

The realisation of truly autonomous systems for the personalised treatment of physiological disorders such as hydrocephalus is closer than ever. This chapter is concerned with the spreading of awareness, particularly among the biomedical engineering community e.g. organisations, companies, physicians and patients, about the possibilities that current technology offers in the area of intelligent and personalised hydrocephalus implants that seek to autonomously manage the symptoms and treat the causes in a manner specifically tuned to the individual patient. This chapter provides an insight into the workings of such a system, its pros and cons and how it can dramatically reduce patient suffering and long hospitalisation periods while increasing the quality of care that is provided.

\subsection{Hydrocephalus}

The human brain is surrounded by a fluid called the cerebrospinal fluid (CSF), that protects it from physical injury, keeps its tissue moist and transports the products of metabolism. This fluid is constantly produced in the parenchyma at rate of approximately $20 \mathrm{ml} . \mathrm{h}^{-1}$ and drained through granulations near the sagittal sinus. If the rate of CSF absorption or drainage is consistently less than the rate of production (for a variety of reasons), the ventricles expand causing the brain to become compressed, leading to the disorder known as hydrocephalus (ASBAH, 2009), as shown in Fig. 1. 


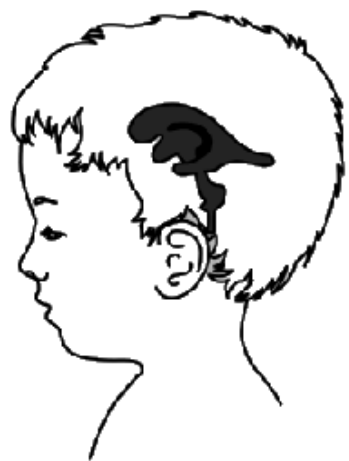

(a)

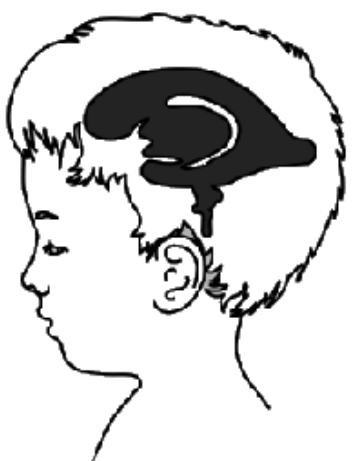

(b)

Fig. 1. Schematic drawing for brain in (a) normal and (b) hydrocephalus cases, showing enlarged ventricles.

This leads to an elevation of the pressure exerted by the cranium on the brain tissue, cerebrospinal fluid, and the brain's circulating blood volume, referred to as intracranial pressure (ICP), and manifest itself in symptoms such as headache, vomiting, nausea or coma. ICP is a dynamic phenomenon constantly fluctuating in response to activities such as exercise, coughing, straining, arterial pulsation, and respiratory cycle. ICP is measured in millimeters of mercury ( $\mathrm{mmHg}$ ) and, at rest, is normally 7-15 $\mathrm{mmHg}$ for a supine adult, and becomes negative (averaging -10 mmHg) in the vertical position (Steiner \& Andrews, 2006). Hydrocephalic patients may experience pressures of up to $120 \mathrm{mmHg}$. If left untreated, elevated ICP may lead to serious problems in the brain.

\subsection{Current Treatment}

Since the 1960s the usual treatment for hydrocephalus is to insert a shunting device in the patient's CSF system. This is simply a device which diverts the accumulated CSF around the obstructed pathways and returns it to the bloodstream, thus reducing ICP, and alleviating the symptoms of hydrocephalus. It consists of a flexible tube with a valve to control the rate of drainage and prevent back-flow.

These valves are passive mechanical devices that open and close depending on either the differential pressure or flow. Although there are various valve technologies and approaches, they all essentially do the same thing, which is to attempt to passively control the symptoms of hydrocephalus by assisting the body's natural drainage system. The valve is usually chosen by the surgeon on the grounds of experience, cost and personal preference.

Despite shunting developments, shunting can have complications, with different types of shunts seemingly associated with different types of complications. Shunt complications can be very serious and become life threatening if not discovered and treated early. However, due to their passive mode of operation, shunt malfunctions are generally not detected before they manifest clinically. These can be divided into issues of under-drainage, over-drainage and infection. Over-drainage and under-drainage are typical drawbacks of such shunts, 
where CSF is either drained in excess or less than needed, which could cause dramatic effects on the patient such as brain damage. The common cause for these two drawbacks might be an inappropriate opening/closing of the valve in respect of the duration or the timing. In other words, valve open for too short/too long periods or it opens/closes at the right timing.

Under-drainage is usually due to blockage of the upper or lower tubes of the shunt by ingrowing tissue, though it can also be caused by the shunt breaking or its parts becoming disconnected from each other. The rate of blockage can be as high as $20 \%$ in the first year after insertion, decreasing to approximately $5 \%$ per year (Casey, et al., 1997), therefore, the clinical presentation of shunt blockage is usually dominated by signs of raised pressure as the brain fluid (CSF) builds up. As ICP is not readily measurable, interferences must be drawn from the symptoms presented. Sometimes the symptoms come on quickly over hour or days, but occasionally they may develop over many weeks with intermittent headache, and tiredness, change in behaviour or deterioration in schoolwork. Diagnosing shunt blockage is not always straightforward. In fact, parents can be as successful at diagnosing shunt blockage as GPs and paediatricians. Whilst additional investigations such as CT scans, $\mathrm{X}$-rays and a shunt taps may help, a definitive diagnosis is sometimes only possible through surgery (ASBAH, 2009).

In the case of over-drainage, the shunt allows CSF to drain from the ventricles more quickly than it is produced. If this happens suddenly, then the ventricles in the brain collapse, tearing delicate blood vessels on the outside of the brain and causing a haemorrhage. This can be trivial or it can cause symptoms similar to those of a stroke. If the over-drainage is more gradual, the ventricles collapse gradually to become slit-like. This often interferes with shunt function causing the opposite problem, high CSF pressure, to reappear. The symptoms of over-drainage can be very similar to those of under-drainage though there are important differences.

Difficulty in diagnosing over-/under-drainage can make treatment of this complication particularly frustrating for patients and their families. It may be necessary to monitor ICP, often over 24 hours. This can be done using an external pressure monitor in the scalp connected to a recorder. Early ICP monitoring is recommended when the clinician is unable to assess the neurological examination accurately. The main concerns are the risks of infection, bleeding, device accuracy and drift of measurement over time. Thus to avoid these risks, a research work is undergoing to develop implanted pressure sensors for short and long term monitoring interrogated by telemetry (Hodgins et al., 2008).

Studies have shown that the use of an 'antisyphon device', a small button inserted into the shunt tubing, will often solve the over-drainage problem, but this does not always work. A 'programmable' or adjustable shunt is intended to allow adjustment of the working pressure of the valve without operation. The valve contains magnets, which allow the setting to be changed by laying a second magnetic device on the scalp. This is undoubtedly useful where the need for a valve of a different pressure arises, but the adjustable valve is no less prone to over-drainage than any other and it cannot be used to treat this condition (Casey et al., 1997). 
One of the obvious reasons for such drawbacks is the inability of such shunts to autonomously respond to the dynamic environment. Inaccuracies and long term drift are also considered among the drawbacks of such shunts. This is mainly due to the fact that these shunts are (typically, but not always) regulated according to the differential pressure across the valves, which differs from intracranial pressure in the brain.

\subsection{Motivations}

Beside their documented drawbacks (Aschoff, 2001; Schley, 2004), shunts do not suit many hydrocephalus patients. This can be realised from the considerable high shunt revision and failure rates (between $30 \%$ and $40 \%$ of all shunts placed in paediatric patients fail within 1 year (Albright et al., 1988; Villavicencio et al., 2003; Piatt et al.,1993; Piatt,1995) and it is not uncommon for patients to have multiple shunt revisions within their lifetime).

Shunt insertion explicitly changes the CSF dynamics in patients with hydrocephalus, causing many to improve clinically. However, the relationship between a changed hydrodynamic state and improved clinical performance is not fully known. Therefore, further research in this area is an important challenge for the hydrocephalus research community, where development of better methods for assessment of CSF dynamic parameters as well as studies to test hypotheses on relationships between CSF dynamics and outcome after shunting is targeted. The aims are for a better understanding of hydrocephalus pathophysiology and to find new predictive tests.

Furthermore, the shunt designers had changed the shunt goal to have the option of reestablishing shunt independence step by step. This means that the statement of Hemmer "once a shunt, always a shunt" is no longer true.

Nevertheless, most patients seem to be only partially shunt-dependent, i.e. their natural drainage system still functions to some extent. The degree of shunt-dependence may range from $1 \%$ to $100 \%$, thus draining $30-50 \%$ of CSF production may be sufficient to keep the ICP within physiological ranges, and only a few need full drainage (Aschoff, 2001). Thus the current generation of shunts do not help patients overcome the underlying problems, but on the contrary, they tend to encourage the patients to become fully shunt dependent. Research has shown however, that in some cases, shunt dependence could be reduced to less than $1 \%$ (Aschoff, 2001) which could even allow the eventual removal of the shunt (Takahashi, 2001). It is envisaged that the next generation of shunts should be able to achieve a controlled shunt arrest in the long run.

The future will bring other options related to the control of CSF production and absorption. Perhaps different valve designs will be more effective in long-term treatment and eventually the development of "smart" shunts. These will be able to react to intracranial physiology and will drain CSF in response to these changes in intracranial dynamics, rather than drain on a continuous basis (Jones \& Klinge, 2008).

To address the lack of personalised treatment, the difficulty in diagnosing shunt faults, the high rate of shunt revisions, the high shunt dependency, and the lack of full understanding 
of shunt effect on the intracranial hydrodynamics, a personalised hydrocephalus shunting system needs to be developed. This would be tasked with the following:

- Frequent non-invasive monitoring of intracranial hydrodynamics to improve treatment outcome.

- $\quad$ Responding to patient symptoms and ICP readings by adjusting treatment.

- Controlling the flow of CSF through a valve of the shunting system.

- Attempting to wean patient off the treatment (shunting system).

- Wirelessly reprogramming the implanted shunting system.

- Instant diagnosis of the shunting system and detection of any fault in the early stages.

By having such system, the hospitalisation periods and patient suffering and inconvenience are reduced, the quality of treatment is improved and better understanding of intracranial hydrodynamics is established thanks to the valuable resource of ICP data.

\subsection{Recent Advances}

In order to achieve such a system, a mechatronic valve is needed which is electrically controlled via software. In this shunting system, the patient could play a vital role in feeding back his/her dissatisfaction, i.e. due to symptoms, regarding the treatment.

In 2005, Miethke claimed patent to a hydrocephalus valve with an electric actuating system comprising a time control system to open and close it (Miethke, 2005). The claim was that such valve would allow improved adaptation to the situation existing in a patient in the case of a hydrocephalus valve.

The intervention of a mechatronic valve provides the opportunity for different shunting systems to be developed. This type of valve can be controlled by software that can vary in its complexity and intelligence. The controlling methods could vary from a simple program that lacks any intelligence to very sophisticated and intelligent program.

Despite ICP monitoring currently being an invasive procedure, patients with hydrocephalus may need repeated episodes of monitoring months or years apart. This is a result of problems arising in which ICP readings are needed for diagnosis. The invasive nature of ICP monitoring has motivated researchers to develop a telemetric implantable pressure sensor for short- and long-term monitoring of ICP with high accuracy (Hodgins et al., 2008). Such sensor was mainly used for monitoring ICP wirelessly by the physician who could manually adjust the valve settings accordingly.

The remainder of the chapter is structured as follows: Section 2 describes the intelligent and personalised shunting system, illustrates its novelty, and lists its functions. In Section 3, the advantages and limitations of the shunting system are identified. Section 4 summaries a quick walkthrough of the shunting system, while Sections 5 and 6 present the future directions and conclusions, respectively. 


\section{Intelligent and Personalised Shunting System}

The new generation of shunting systems are expected to overcome the drawbacks and limitations of the current shunting systems. A novel intelligent telemetric system is developed for the improved management and treatment of hydrocephalus. The intelligent system would autonomously manage the CSF flow, personalise the management of CSF flow through involving real-time intracranial pressure readings and patient's feedback, and responding to them. It also would autonomously manage and personalise the treatment of hydrocephalus, thus providing treatment that is personalised, goal-driven and reactive as well as pro-active, which gradually reduce shunt dependence and eventually establish a controlled arrest of the shunt. In addition, it would be able to monitor performance of its components, thus minimising the shunt revisions, and establish distant treatment database (e.g. computer-based patient record) and exchange treatment information, by regularly reporting the patient's record to the physician.

All these qualities can only be attained by a multi-agent approach (Momani, et al., 2008). This would also involve replacing a passive valve (commonly used in hydrocephalus shunts) with a mechatronic valve controlled by an intelligent microcontroller that wirelessly communicates with a separate smart hand-held device. The system is illustrated in Fig. 2.

This shunting system would consist of two subsystems; implantable and external (patient device). The implanted subsystem would mainly consist of ultra low power commercial microcontroller, mechatronic valve, pressure sensor and low power transceiver. This implantable shunting system would wirelessly communicate with a hand-held smartphone operated by the patient, or on the patient's behalf by a clinician or guardian. This device would have a graphical user interface and an RF interface to communicate with the user and the implantable wireless shunt respectively.

This system would also enable a physician to monitor and modify the treatment parameters wirelessly, thus reducing, if not eliminating, the need for shunt revision operations. Once implanted, such a system could lead not only to better treatment of the users of such shunts, but also allow the dynamics of this disease and the effect of shunting to be understood in greater depth.

An intelligent system, e.g. (Momani et al., 2008) , can be used to autonomously regulate the mechatronic valve according to a time-based schedule and update it based on the intracranial pressure that is measured when needed. In such system, ICP readings and other sensory inputs such as patient feedback would help in tuning the treatment and enabling the intervention of the medical practitioner to update and manually adapt the schedule. This would result in a personalised and intelligent CSF management, which leads to every patient having different management schedule according to his/her personal conditions.

\subsection{Novelty}

The idea of using a pressure sensor integrated into a shunt system for monitoring ICP and interrogated by telemetry is not in itself a novel idea (Ginggen, 2007; Jeong et al., 2004; Miesel \& Stylos, 2001), where ICP readings used by the physician to monitor the 


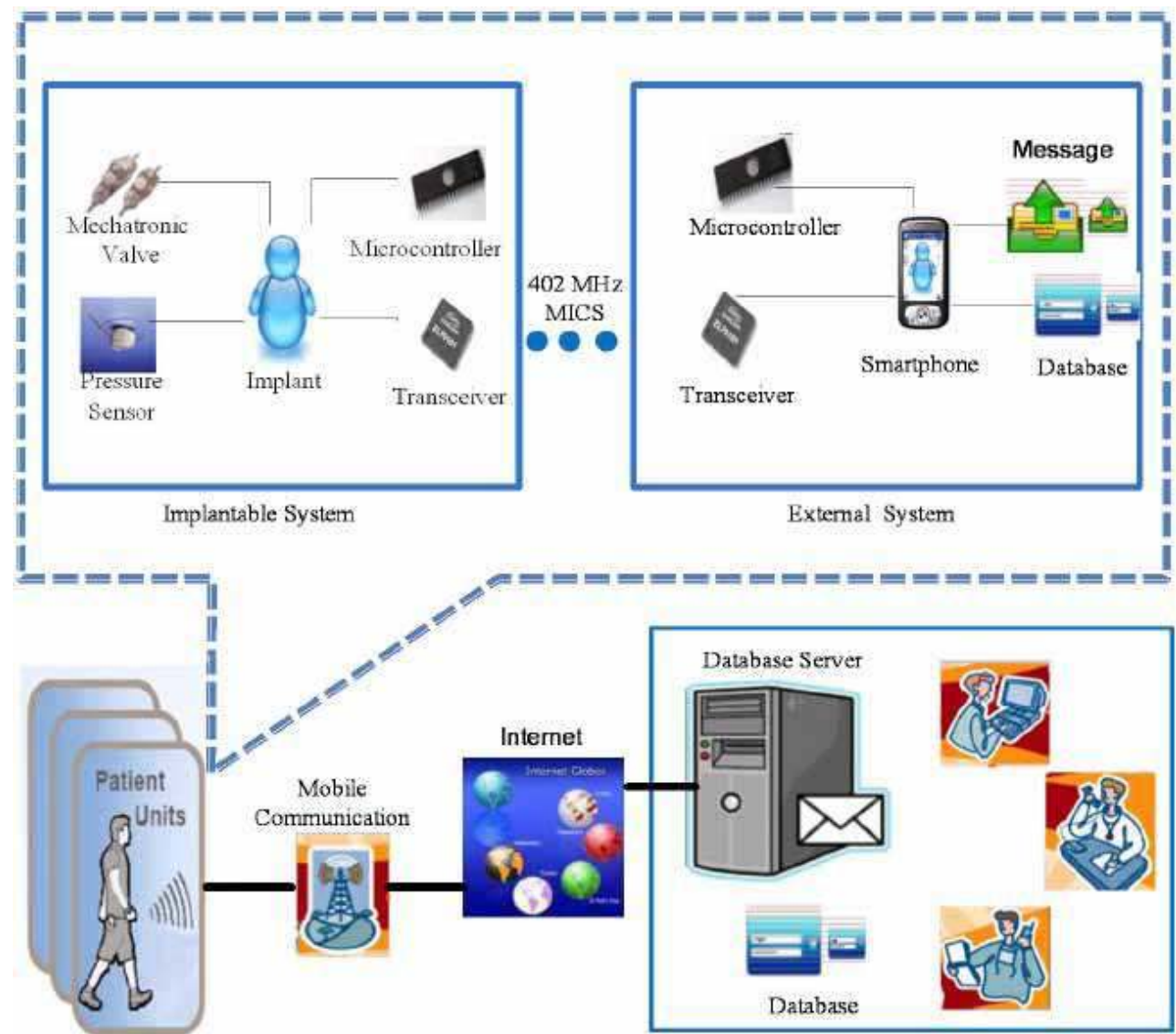

Medical Centre

Fig. 2. Schematic diagram of the intelligent and personalised shunting system.

performance of the implanted shunt. However, the novelty in this work is in having an implantable shunting system that utilise these readings in addition to patient input as a direct feedback to instantaneously and even autonomously manage the shunt, i.e. analyse the feedback, diagnose any shunt faults and accordingly regulate the opening of a mechatronic valve. Thus an element of intelligence and personalisation would be added to the mechatronic shunting system by enabling real-time reconfiguration of the shunt parameters based on the patient's response and the ICP readings.

\subsection{Strategy and Approach}

The mechatronic valve is controlled by a time based schedule. The schedule would be simply the distribution of the valve state (open/close) over time. Such schedule would incur many disadvantages e.g. over-/under-drainage, if its selection is arbitrary. In order to optimise the usefulness of such a valve, its schedule should be selected in way that delivers a personalised treatment for each specific patient. Achieving such a goal is not an easy task 
due to the dynamic behaviour of intracranial pressure that not only varies among patients but also within individual patient with time. There are two extremes for schedule alternatives. One is a dynamic schedule that responds to the instantaneous intracranial pressure which requires an implanted pressure sensor, i.e. closed loop shunting system. The other extreme is a fixed schedule that has a fixed open frequency over 24 hours. This alternative lacks flexibility and ignores the intracranial dynamic behaviour while the first is impractical.

A schedule structure is proposed that offers a compromise between the two schedule extremes. Thus to facilitate the process of schedule selection and to add some degree of flexibility, a 24-hours schedule, shown in Fig. 3, is divided into 24 one hour sub-schedules. Each sub-schedule is identified by three parameters; the targeted hour (hr), open duration $\left(d_{O N}\right)$ and closed duration $\left(d_{O F F}\right)$ for that specific hour.

Treatment in the proposed shunting system is presented by a time-based valve schedule, thus dynamically modifying the schedule, would mean changing the applied treatment. Treatment would be modified in order to adapt to the individual patient and actual conditions. This modification is accomplished based on real-time inputs (e.g. symptoms delivered via patient feedback and internally measured ICP) and derived parameters such as rate of ICP change, effective opening time and figure of merits. To update the schedule, the modification is only applied on the targeted sub-schedule (hour).

\begin{tabular}{|c|c|c|c|c|}
\hline 1 & 2 & 3 & $\ldots \ldots$ & 24 \\
\hline$\left(\mathrm{d}_{\mathrm{ONL}} \mathrm{d}_{\mathrm{Off} 1}\right)$ & $\left(\mathrm{d}_{\mathrm{ON2} 2}, \mathrm{~d}_{\mathrm{O}+\mathrm{f} 2 \mathrm{f}}\right)$ & $\left(\mathrm{d}_{\mathrm{ON} 3}, \mathrm{~d}_{\mathrm{OfF}+3}\right)$ & $\ldots \ldots$ & $\left(d_{0 N 24}, d_{0 f F 24}\right)$ \\
\hline$\square \square$ & 口几 & & ..... & $\square$ \\
\hline
\end{tabular}

Fig. 3. A 24-hour schedule for the implanted valve.

The system acquires knowledge directly and wirelessly from the patient's satisfaction input (feedback), to make decision regarding modifying the schedule or it just records and saves patient's satisfaction for future interpretation.

Once the shunting system is implanted, the system is initially programmed by taking into consideration the empirical data patient's history, e.g. ICP data, personal information, medical history, family history.

In long run, the system should become stable and reach a state in which it adapts to the patient and deals smartly and dynamically with any changes with no need for help. As a result, these personalised schedules can be categorised according to hydrocephalus patient types so as to develop an optimum schedule for each patient's category that can be used, in future, as the initial schedule when implanting such shunts.

\subsection{Functions}

The intelligent shunting system will perform two main roles; management and treatment. 


\subsubsection{Management}

This involves managing both the physiological condition and the shunting system itself. The former consists of monitoring and optimising the success of treatment, adapting the treatment to the individual and actual conditions, responding to symptoms reported via patient feedback and capturing real shunt dependency. On the other hand the latter covers the self monitoring, diagnosis and fault detection. Both of those aspects are detailed below.

\section{A. Managing Hydrocephalus}

Similar to any other shunt, the proposed shunt will aim to control ICP within the normal physiological limits. To achieve this, the following tasks are performed,

1. Monitoring the success of treatment and its optimisation: The shunt will routinely collect ICP readings measured by the implanted sensor, analyse them internally to check whether the current schedule succeeded in maintaining pressure within normal range. In addition, a figure of merit is calculated to help in evaluating the performance of treatment and in selecting a schedule that best suit the situation. The novelty of such function would be in it is ability to collect ICP data while the valve is closed, thus providing a valuable record of ICP for un-shunted case (without treatment) with no need to perform any additional invasive operation. Such traces are considered valuable in understanding specific-patient cases and the effect of applying different schedules, since currently physician do not perform ICP monitoring before shunting unless all other methods did not work out in diagnosing hydrocephalus due to the risks of such procedure.

2. Adapting the treatment to the individual and actual conditions: to successfully manage hydrocephalus, it should adapt the treatment to the needs of the specificpatient and arising circumstances. If a problem arises in the measured ICP (e.g. ICP is high), the system would respond dynamically and instantaneously by updating the valve schedule according to rules saved in the knowledge base. Initially these rules are general but with time it is revised by the shunting system to suit this particular patient.

3. Responding to symptoms delivered via patient feedback: Nowadays, reoccurrence of symptoms in shunted patient is usually dealt by externally monitor the ICP. Such procedure is invasive and accompanied by many risks and complications. That is why intracranial monitoring usually is the last option for un-shunted patient unless it is vital to diagnose hydrocephalus in some cases. In this system, patient feedback would be logged into the patient device to represent the type of symptom and its severity. As a result of receiving such feedback, the shunting system will investigate the cause of the symptom by checking the normality of ICP and perform self-checking for any faults in the system. And later draw a conclusion whether the cause was due to abnormality in ICP or not. In the case of any abnormality, it will respond by either modifying the valve schedule to accommodate the symptom or alerting the physician in case of faults possibilities. 
The availability of such option in the proposed shunting system, spares patient from unnecessary pain, suffering and risks accompanied with the current diagnosis method. And on the contrary to current methods, this option will provide an instant diagnosing while the patient is living his/her normal life, thus no need to wait for an appointment or being hospitalised

4. Capturing real shunt dependency: Knowing that patients seem to be only partially shunt-dependent, the current shunts do not help in revealing the degree of dependency, but on the contrary, they tend to encourage the patients to become fully shunt dependent. Proposed shunting system can help in revealing the actual shunt dependency, thus allowing the natural drainage to keep working at its maximum power and the shunt will only give a hand when the natural drainage is overloaded.

\section{B. Managing the Shunting System}

It is important that the system functions properly so that a reasonable intracranial pressure is maintained. Currently, shunt faults are the leading cause of shunt revisions. The main shunt faults are blockage and disconnection. In an effort to detect these faults in early stages, thus avoiding any further patient inconveniences that could arise if left undetected, the proposed shunting system will perform the following preventive procedure.

1. Self monitoring: routinely check up if the ICP data changes in responsive manner to the valve states.

2. Self diagnosis: use novel fault detection measures, which are based on ICP data and valve status, to find any possibility of occurrence of any fault, determine its type (e.g. shunt blockage/disconnection/breakage or sensor dislocation/drift), and its degree.

3. Power management: use a real-time self wake-up method to manage the power consumption in the implanted shunt.

4. Memory management: use a novel method to reduce the size of stored data in the implanted shunt, thus solving a problem associated with implanted memory limitations.

\subsubsection{Treatment}

The goal of shunting has changed over time since it was first used. The shunt nowadays is expected to provide an option of establishing gradual shunt arrest. It is also the dream of any hydrocephalus shunted patient to regain his/her independence of the shunt and mainly rely on his/her reconditioned natural drainage system.

The capability of the proposed system to be wirelessly reprogrammed without the need for surgery and its ability to monitor the change in the intracranial hydrodynamics are essential in facilitating the shunt arrest process.

At the stage when the shunting system is fully in control of the intracranial hydrodynamics and the patient's real shunt dependency is captured, the shunting system will start achieving new objective that is reducing shunt dependency and might eventually arrest the use of the shunt (weaning). 
The weaning process will involve manipulating two parameters; the length of open duration and the limits of acceptable pressure (above which ICP is considered abnormal), in away that make the patient either adapt gradually to higher level of ICP or reactivate the natural drainage to take part of the drainage process. Weaning will be implemented over stages. The length of each stage will vary based on patient response and capability to accommodate such change. For each weaning stage, the effect of modifying weaning parameters will be evaluated by routinely collecting ICP readings and patient feedback. The amount of reduction in the open duration or increase in the acceptable pressure limits will depend on parameters derived from patient's ICP data at different valve states.

\section{Advantages and Limitations}

The shunting system is explored and its advantages are identified. Furthermore, limitations facing implementing such system are investigated.

\subsection{Advantages}

Compared to the current shunts, this shunting system offers the following advantages:

- Personalising: responsive to patient needs and situation.

- Autonomous: functions without supervision or intervention.

- Reducing patient suffering, e.g. hospitalisation.

- Managing and responding to symptoms obtained from patient feedback.

- Autonomous monitoring and diagnosis of intracranial hydrodynamics.

- Potential to achieve arrest of shunt dependence.

- Wireless reprogramming; access, modify and replace current parameters.

- Ability to obtain ICP traces for patient both with and without shunt.

- Shunt self diagnosis and fault detection.

- Better understanding of hydrocephalus, intracranial hydrodynamics and the effect of shunting on them.

\subsection{Limitations}

The following limitations are encountered when implementing such system:

- ICP sensor inaccuracy or breakage

- Mechatronic valve intermittent problems

- Physician and patient mentality

- Technical issues

- Power limitation

- Implantable memory size limitation

- Product size limitation

- Potential faults

\section{Walkthrough}

A quick walk through the shunting system is summarised. It illustrates the shunt functions through an example of one day in the life of shunted hydrocephalus patient. 
Bob is a hydrocephalus patient. Today, he was shunted with an intelligent shunting system. This system has been configured by the physician to suit Bob based on his medical history (including an ICP trace) and hydrocephalus type.

Once implanted, the system will attempt to initialise itself by first collecting ICP data for 24 hours and then instantiate an initial personalised 24 sub-schedules based on hourly derived parameters (e.g. average ICP and rate of change in ICP) from the collected data. Starting from the first day, the implanted shunt will perform its routine tasks; ICP monitoring, valve regulating according to the schedule, self diagnosis, and daily backup of the results.

One day Bob woke up and he was feeling drowsy. He checked if there were any alerts on his patient device (PD) but found nothing. He started to worry that there might be a problem with his shunt, thus he logged his feedback on his PD.

In the following few minutes, the intelligent agent on PD started to investigate the cause by firstly sending a request for ICP data to the implanted shunt. While waiting for a reply, it checked its database if any similar feedback that might have occurred previously at the time of the day or if such symptom is recently reoccurring.

Meanwhile, the implanted shunting system received the request and immediately initiated the ICP sensor to collect data over a period of time at different valve states. As soon as sufficient data is collected, it is sent wirelessly to the PD.

By receiving the ICP, the external shunting system (PD) starts performing analysis and calculating some derived parameters to check if the cause for such symptoms is due ICP abnormality or shunt fault. If the results of the analysis indicated that the cause of the symptom is not due to ICP abnormality or shunt fault, then a message will show up on the PD display to reassure Bob that the symptom is not ICP-related. The feedback, its time along with the ICP data and the decision made are saved to be uploaded at a later time to Bob's personal record in the central database at the hospital. On the other hand, if the results showed that the cause is due to ICP abnormality, then the intelligent system will work on modifying the schedule at that hour and track its effect for the next couple of days. A message will also show up telling Bob that the problem has been handled. Bob in either case was reassured that his shunting system was functioning properly and there was nothing to worry about.

While Bob is doing his job, the implanted shunt is regulating the valve according to a timebased schedule and at the same time perform a check up on the ICP and the shunt itself. To do this, it collects ICP data while the valve is open and closed. It checks if these data is within the acceptable limits and if not, it will alert the PD to perform modification on the schedule. The implanted shunt will also calculate some derived parameter to detect any possibility of fault occurrence in the shunt. In case any fault is detected, the implanted shunt will inform the physician through the $\mathrm{PD}$, in order to take some procedures in early stage to spare Bob from unnecessary pain and suffering. 
After one year of shunt experience, Bob confidence in his shunt has grown and he stopped worrying about his ICP since he knows that wherever he is, he has a personal physician that accompanies him 24 hours a day and whom will worry on his behave. Bob also pleased that he no longer has to wait for an appointment or stay in the hospital every time he had a symptom. He can now check up his ICP and shunt in minutes while having his normal life anywhere and anytime.

Two years passed on the shunting surgery. Bob is happy with his shunt, he has not experienced any symptom for long time. Thus, his shunt has recognised this progress and decided after consulting the physician to start reducing shunt dependency (shunt weaning process). First step was to reduce open duration for a selected hour based on Bob's ICP history. Bob is asked to play a vital role at this stage, by giving his feedback whenever he has symptoms, to tune and personalise the weaning process. After checking that the first step did not have harmful consequences, the shunt proceeded to its second step which is attacking a new open duration and try to reduce it. Unfortunately this time Bob could not handle the severity of the symptoms thus the shunt had to reconfigure this step to avoid any inconvenience for Bob.

After prolong period of time, Bob's shunt dependency has been reduced to minimum but unfortunately Bob's brain adaptability could not go through a complete shunt arrest. Nevertheless, Bob was really satisfied with what his shunt has done and what he is still doing and hopes that shunt arresting can be achieved in later time.

\section{Future Directions}

Future enhancements would include incorporating more parameters in developing and modifying the valve schedule. For example, patient daily activities (sleeping and working times, type of work (sitting, standing)) and other parameters derived from ICP traces would enhance the performance of the valve schedule if taken into consideration when deriving or modifying a schedule.

The significance of such intelligent personalised shunting system can be extended by incorporating it into a distributed network of intelligent shunts, where data mining and knowledge acquisition techniques are deployed to analyse and interpret hydrocephalus patients' data for case enquiring, treatment plan advising, and ICP classification and patient clustering. In addition it would let patients exchange and share the treatment and management process.

\section{Conclusion}

The realisation of truly autonomous shunting systems for personalised hydrocephalus treatment is closer than ever. This requires the use of an implanted mechatronic valve and pressure sensor, a smart hand held device, improved algorithms to analyse the inputs (e.g. ICP readings and patient feedback) and extract relevant information from raw data, and rule-based decisions controlled by local intelligence. The Management of intracranial hydrodynamics, shunt self-diagnosis, and treatment of hydrocephalus can be continuously 
and autonomously monitored and parameters changed as necessary by the intelligent software in the handheld device via wireless communication and data will be sent on demand to the clinician for further evaluation. Such shunting system would give hydrocephalus patients the freedom to go anywhere they like while receiving medical services and health care in a timely fashion. Visits of patients to hospitals or the doctor will be reduced to a necessary minimum, while increasing the quality of care that is provided.

\section{References}

Association for Spina Bifida Hydrocephalus (2009). Hydrocephalus, available online: http://www.asbah.org/.

Albright, A.L.; Haines, S.J. \& Taylor, F.H. (1988). Function of parietal and frontal shunts in childhood hydrocephalus, J Neurosurg, vol. 69, pp. 883-886.

Aschoff, A. (2001). The evolution of shunt technology in the last decade: A critical review, presented at 3rd International Hydrocephalus Workshop, Kos, Greece, May 17-20th, 2001.

Casey, A. T.; Kimmings, E. J.; Kleinlugtebeld, A. D.; Taylor. W. A.; Harkness, W. F. \& Hayward, R. D. (1997). The long-term outlook for hydrocephalus in childhood. (A ten-year cohort study of 155 patients), Pediatr Neurosurg, vol. 27, no. 2, pp. 63-70.

Ginggen, A. (2007). Optimization of the Treatment of Hydrocephalus by the Non-Invasive Measurement of the Intra-Cranial Pressure, PhD thesis, infoscienc, EPFL, Czech. URL : http:/ /library.epfl.ch/theses/?nr=3757.

Hodgins, D.; Bertsch, A.; Post, N.; Frischholz, M.; Volckaerts, B.; Spensley, J.; Wasikiewicz, J. M.; Higgins, H.; Stetten, F. \& Kenney, L. (2008). IEEE Pervasive Computing. vol. 7, no. 1, pp. 14-21, January-March 2008.

Jones, H. C. \& Klinge, P. T. (2008). Hydrocephalus, In Hannover Conference 17-20th September 2008, Cerebrospinal Fluid Res., 2008; vol. 5, pp. 19.

Jeong, J. S. ; Yang, S. S.; Yoon, H. J. \& Jung, J. M. (2004). Micro Devices for a Cerebrospinal Fluid (CSF) Shunt System. Sensors and Actuators A, Vol. 110, pp. 68-76.

Kramer, L. C.; Azarow, K.; Schlifka, B. A. \& Sgouros, S. (2006). eMedicine Pediatrics, available online: http://emedicine.medscape.com/article/937979-overview.

Miesel, K. A. \& Stylos, L. (2001). Intracranial monitoring and therapy delivery control device, system and method, United States Patent, No. 6248080.

Miethke, C. (2005). Hydrocephalus valve, U.S. Patent 6926691, August 9, 2005.

Momani, L., Alkharabsheh, A. \& Al-Nuaimy, W. (2008). Design of an intelligent and personalised shunting system for hydrocephalus, in Conf Proc 2008 IEEE Eng Med Biol Soc., Vancouver, Canada, pp. 779-782.

Piatt Jr, J.H. \& Carlson, C.V. (1993). A search for determinants of cerebrospinal fluid shunt survival: retrospective analysis of a 14-year institutional experience. Pediatr Neurosurg, vol. 19, pp. 233-241.

Piatt Jr, J.H. (1995). Cerebrospinal fluid shunt failure: late is different from early. Pediatr Neurosurg, vol. 23, pp. 133-139.

Schley, D.; Billingham, J. \& Marchbanks, R. J. (2004). A Model of in-vivo hydrocephalus shunt dynamics for blockage and performance diagnostics, Mathematical Medicine and Biology, vol. 21, no. 4, pp. 347-368, Dec. 2004. 
Steiner, L. A. \& Andrews, P. J. (2006). Monitoring the injured brain: ICP and CBF, British Journal of Anaesthesia, vol. 97, no. 1 (July 2006), pp. 26-38.

Takahashi, Y. (2001). Withdrawal of shunt systems clinical use of the programmable shunt system and its effect on hydrocephalus in children, Childs Nerv Syst, vol. 17, pp. 472-477, Aug. 2001.

Villavicencio, A. T.; Leveque, J.; McGirt, M. J.; Hopkins, J. S.; Fuchs, H. E. \& George, T. M. (2003). Comparison of Revision Rates Following Endoscopically Versus Nonendoscopically Placed Ventricular Shunt Catheters, Surgical Neurology, vol. 59, no. 5, pp. 375-379. 


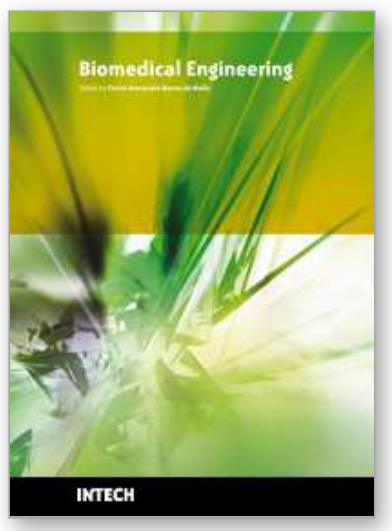

\author{
Biomedical Engineering \\ Edited by Carlos Alexandre Barros de Mello
}

ISBN 978-953-307-013-1

Hard cover, 658 pages

Publisher InTech

Published online 01, October, 2009

Published in print edition October, 2009

Biomedical Engineering can be seen as a mix of Medicine, Engineering and Science. In fact, this is a natural connection, as the most complicated engineering masterpiece is the human body. And it is exactly to help our "body machine" that Biomedical Engineering has its niche. This book brings the state-of-the-art of some of the most important current research related to Biomedical Engineering. I am very honored to be editing such a valuable book, which has contributions of a selected group of researchers describing the best of their work. Through its 36 chapters, the reader will have access to works related to ECG, image processing, sensors, artificial intelligence, and several other exciting fields.

\title{
How to reference
}

In order to correctly reference this scholarly work, feel free to copy and paste the following:

Lina Momani, Abdel Rahman Alkharabsheh and Waleed Al-Nuaimy (2009). Intelligent and Personalised Hydrocephalus Treatment and Management, Biomedical Engineering, Carlos Alexandre Barros de Mello (Ed.), ISBN: 978-953-307-013-1, InTech, Available from: http://www.intechopen.com/books/biomedicalengineering/intelligent-and-personalised-hydrocephalus-treatment-and-management

\section{INTECH}

open science | open minds

\section{InTech Europe}

University Campus STeP Ri

Slavka Krautzeka 83/A

51000 Rijeka, Croatia

Phone: +385 (51) 770447

Fax: $+385(51) 686166$

www.intechopen.com

\section{InTech China}

Unit 405, Office Block, Hotel Equatorial Shanghai

No.65, Yan An Road (West), Shanghai, 200040, China

中国上海市延安西路65号上海国际贵都大饭店办公楼405单元

Phone: +86-21-62489820

Fax: +86-21-62489821 
(C) 2009 The Author(s). Licensee IntechOpen. This chapter is distributed under the terms of the Creative Commons Attribution-NonCommercial-ShareAlike-3.0 License, which permits use, distribution and reproduction for non-commercial purposes, provided the original is properly cited and derivative works building on this content are distributed under the same license. 